PROCEEDINGS OF THE

AMERICAN MATHEMATICAL SOCIETY

Volume 138, Number 6, June 2010, Pages 2099-2105

S 0002-9939(10)10262-7

Article electronically published on January 27, 2010

\title{
CONVEXITY OF HESSIAN INTEGRALS AND POINCARÉ TYPE INEQUALITIES
}

\author{
ZUOLIANG HOU \\ (Communicated by Chuu-Lian Terng)
}

\begin{abstract}
In this paper, integrals involving both real and complex Hessian operators over bounded domains are studied. Poincaré type inequalities are proved in both cases, which generalizes an early result of Trudinger and Wang.
\end{abstract}

\section{INTRODUCTION}

Throughout this paper, $k$ and $n$ will be two fixed integers such that $0 \leq k \leq n$. For $\lambda \in \mathbb{R}^{n}$, let $S_{k}(\lambda)$ be the normalized $k^{\text {th }}$ elementary symmetric polynomial of $\lambda$, i.e.

$$
S_{k}\left(\lambda_{1}, \cdots, \lambda_{n}\right)=\frac{k !(n-k) !}{n !} \sum_{i_{1}<i_{2}<\cdots<i_{k}} \lambda_{i_{1}} \cdots \lambda_{i_{k}} .
$$

Let $\mathcal{H}(n)$ denote the space of Hermitian matrices of size $n$. For $A \in \mathcal{H}(n)$, let $\lambda(A) \in \mathbb{R}^{n}$ denote the eigenvalue of $A$ and

$$
S_{k}(A)=S_{k}(\lambda(A)) \text {. }
$$

Even though we use $S_{k}$ to denote functions defined on both $\mathbb{R}^{n}$ and $\mathcal{H}(n)$, its meaning should be clear from the context. In either case, we use $\tilde{S}_{k}$ to denote the complete polarization of $S_{k}$. Let

$$
\Gamma_{k}=\left\{\lambda \in \mathbb{R}^{n} \mid S_{j}(\lambda) \geq 0, j=1, \ldots, k\right\}
$$

be the $k$-positive cone in $\mathbb{R}^{n}$.

Let $\Omega$ be a smooth bounded domain in $\mathbb{C}^{n}$. For a real-valued function $u \in \mathcal{C}^{2}(\Omega)$, we define the complex $k$-Hessian of $u$ by

$$
H_{k}[u]=S_{k}\left(\operatorname{Hess}_{\mathbb{C}}(u)\right),
$$

where $\operatorname{Hess}_{\mathbb{C}}(u)$ is the complex Hessian matrix of $u$. Let

$$
\mathcal{P}_{k}(\Omega)=\left\{u \in \mathcal{C}^{2}(\bar{\Omega}) \mid \lambda\left(\operatorname{Hess}_{\mathbb{C}}(u)\right) \in \Gamma_{k}\right\}
$$

be the space of $k$-plurisubharmonic ( $k$-psh) functions and let $\mathcal{P}_{k}^{0}(\Omega)$ be the subspace of $\mathcal{P}_{k}(\Omega)$ containing functions vanishing on $\partial \Omega$. It is well known Går59 that for $v_{j} \in \mathcal{P}_{k}(\Omega), j=1, \cdots, k$,

$$
\tilde{H}_{k}\left[v_{1}, \cdots, v_{k}\right]=\tilde{S}_{k}\left(\operatorname{Hess}_{\mathbb{C}}\left(v_{1}\right)\right), \cdots, \lambda\left(\operatorname{Hess}_{\mathbb{C}}\left(v_{k}\right)\right) \geq 0 .
$$

Received by the editors May 25, 2009, and, in revised form, September 27, 2009.

2010 Mathematics Subject Classification. Primary 39B62; Secondary 26D10.

Key words and phrases. Complex Hessian, energy functional.

(C)2010 American Mathematical Society Reverts to public domain 28 years from publication 
For $u, u_{0}, \cdots, u_{k} \in \mathcal{C}^{2}(\bar{\Omega})$, denote

$$
F_{k}\left[u_{0}, \cdots, u_{k}\right]=\int_{\Omega}\left(-u_{0}\right) \tilde{H}_{k}\left[u_{1}, \cdots, u_{k}\right]
$$

and

$$
I_{k}[u]=F_{k}[u, \cdots, u]=\int_{\Omega}(-u) H_{k}[u] .
$$

Theorem 1. With the notation as above:

(a) For any $u_{0}, \cdots, u_{k} \in \mathcal{P}_{k}^{0}(\Omega)$,

$$
F_{k}\left[u_{0}, \cdots, u_{k}\right] \leq \prod_{j=0}^{k}\left(I_{k}\left[u_{j}\right]\right)^{\frac{1}{k+1}} .
$$

(b) For a fixed integer $m$ such that $0 \leq m<k$ and fixed $v_{1}, \cdots, v_{m} \in \mathcal{P}_{k}(\Omega)$, consider the functional

$$
\mathcal{P}_{k}^{0}(\Omega) \ni u \mapsto I_{k, v_{1}, \cdots, v_{m}}[u]=\int_{\Omega}(-u) \tilde{H}_{k}\left[u, \cdots, u, v_{1}, \cdots, v_{m}\right] .
$$

Then $I_{k, v_{1}, \cdots, v_{m}}^{1 /(k-m+1)}$ is convex on $\mathcal{P}_{0}^{k}$ in the sense that for any $u, v \in \mathcal{P}_{k}^{0}(\Omega)$,

$$
I_{k, v_{1}, \cdots, v_{m}}^{\frac{1}{k-m+1}}[u+v] \leq I_{k, v_{1}, \cdots, v_{m}}^{\frac{1}{k-m+1}}[u]+I_{k, v_{1}, \cdots, v_{m}}^{\frac{1}{k-m+1}}[v] .
$$

Recall that a smooth bounded domain $\Omega$ is strongly $k$-pseudoconvex if $\partial \Omega$ is connected and the $(k-1)^{\text {th }}$ elementary symmetric polynomial of the eigenvalues for the Levi form of $\partial \Omega$ is strictly positive on $\partial \Omega$. For a strongly $k$-pseudoconvex domain, we have the following Poincaré type inequalities.

Theorem 2. Suppose $\Omega$ is strongly $k$-pseudoconvex. Then for $0 \leq m<k$, there exists a constant $C$ such that for any $u \in \mathcal{P}_{k}^{0}(\Omega)$,

$$
\left(I_{m}[u]\right)^{\frac{1}{m+1}} \leq C\left(I_{k}[u]\right)^{\frac{1}{k+1}}
$$

where the constant $C$ depends on $m, k$ and $\Omega$ only.

Some special cases of Theorem 1 were well known. For example, Cegrell and Persson proved in [CP97] that if both $u$ and $v$ are psh functions on a domain with vanishing boundary data, then for an integer $j$ such that $0 \leq j \leq n$,

$$
\int_{\Omega}(-u)\left(d d^{c} u\right)^{j}\left(d d^{c} v\right)^{n-j} \leq\left(\int_{\Omega}(-u)\left(d d^{c} u\right)^{n}\right)^{\frac{j+1}{n+1}}\left(\int_{\Omega}(-v)\left(d d^{c} v\right)^{n}\right)^{\frac{n-j}{n+1}} .
$$

This is just a special case of equation (11) with $u_{0}=\cdots=u_{j}=u$ and $u_{j+1}=\cdots=$ $u_{n}=v$.

Since the proof of Theorem 1 uses only the divergence structure of the Hessian operator $H_{k}$, it can also be applied to the study of real Hessian operators and integrals. Let $D$ be a smooth bounded domain in $\mathbb{R}^{n}$. For a real-valued function $u_{j} \in \mathcal{C}^{2}(\bar{D}), j=0,1, \cdots, k$ and $u \in \mathcal{C}^{2}(\bar{\Omega})$, we define

$$
G_{k}\left[u_{0}, \cdots, u_{k}\right]=\int_{D}\left(-u_{0}\right) \tilde{S}_{k}\left[\operatorname{Hess}\left(u_{1}\right), \cdots, \operatorname{Hess}\left(u_{k}\right)\right]
$$

and

$$
J_{k}[u]=G_{k}[u, \cdots, u]
$$

where $\operatorname{Hess}(u)$ is the Hessian matrix of $u$. 
Theorem 3. For any $k$-convex function $u_{0}, \cdots, u_{k}$ with $u_{0}=\cdots=u_{k}=0$ on $\partial D$,

$$
G_{k}\left[u_{0}, \cdots, u_{k}\right] \leq \prod_{j=0}^{n}\left(J_{k}\left[u_{j}\right]\right)^{\frac{1}{k+1}} .
$$

Theorem 4. Suppose $D$ is a smooth bounded domain in $\mathbb{R}^{n}$ such that $S_{k-1}(I I)>0$ on $\partial D$, where $I I$ is the second fundamental form of $\partial D$. Then for an integer $m$ with $0 \leq m<k$, there exists a constant $C$ depending on $m, k$ and $D$ only such that

$$
\left(J_{m}[u]\right)^{\frac{1}{m+1}} \leq C\left(J_{k}[u]\right)^{\frac{1}{k+1}}
$$

for any $k$-convex function $u$ with $u=0$ on $\partial D$.

Theorem 4 was originally proved by Trudinger and Wang in TW98 using the parabolic method. Compared with their proof, our proof is quicker and more elementary and can be adopted to study the complex Hessian integral. On the other hand, Trudinger and Wang's proof contains more information. They showed that the best constant $C$ in equation (5) is attained by the solution of the Dirichlet problem $H_{k}[u]=H_{m}[u]$ and $u=0$ on $\partial D$.

Hessian integrals such as $J_{k}$ were first studied by Bakelman Bak83 and Tso Tso90 in the setting of a real Monge-Ampère operator. In Wan94, Wang studied the real Hessian integrals for general $k$ and established some Sobolev type inequalities. For more information on the real Hessian integral, see Wan09] and the references therein.

For simplicity, we always assume that the functions studied in this paper are at least twice differentiable. By an appropriate approximation, it is possible to extend the results to the case of real and complex Hessian measures for nonsmooth functions; see [TW02, Bło05] for more details.

\section{Complex Hessian integrals}

Let $\omega$ be the Kähler form of the standard Euclidean metric of $\mathbb{C}^{n}$, i.e.

$$
\omega=\sqrt{-1} \sum_{j=1}^{n} d z^{j} \wedge d \bar{z}^{j} .
$$

It is easy to check that for $u, u_{0}, \cdots, u_{k} \in \mathcal{P}_{k}(\Omega)$,

$$
H_{k}[u] \omega^{n}=(\sqrt{-1} \partial \bar{\partial} u)^{k} \wedge \omega^{n-k}
$$

and

$$
\tilde{H}_{k}\left[u_{1}, \cdots, u_{k}\right] \omega^{n}=\left(\sqrt{-1} \partial \bar{\partial} u_{1}\right) \wedge \cdots \wedge\left(\sqrt{-1} \partial \bar{\partial} u_{k}\right) \wedge \omega^{n-k} .
$$

Hence

$$
I_{k}[u]=\int_{\Omega}(-u)(\sqrt{-1} \partial \bar{\partial} u)^{k} \wedge \omega^{n-k}
$$

and

$$
F_{k}\left[u_{0}, \cdots, u_{k}\right]=\int_{\Omega}\left(-u_{0}\right)\left(\sqrt{-1} \partial \bar{\partial} u_{1}\right) \wedge \cdots \wedge\left(\sqrt{-1} \partial \bar{\partial} u_{k}\right) \wedge \omega^{n-k}
$$


Obviously the $F_{k}$ in equation (6) is symmetric in $u_{1}, \cdots, u_{k}$. If $u_{j} \in \mathcal{P}_{k}^{0}(\Omega)$ for $j=0, \cdots, k$, then by integration by parts,

$$
\begin{aligned}
F_{k}\left[u_{0}, \cdots, u_{k}\right] & =\int_{\Omega} \sqrt{-1} \partial u_{0} \wedge \bar{\partial} u_{1} \wedge \cdots \wedge\left(\sqrt{-1} \partial \bar{\partial} u_{k}\right) \wedge \omega^{n-k} \\
& =\int_{\Omega}\left(-u_{1}\right)\left(\sqrt{-1} \partial \bar{\partial} u_{0}\right) \wedge \cdots \wedge\left(\sqrt{-1} \partial \bar{\partial} u_{k}\right) \wedge \omega^{n-k} \\
& =F_{k}\left[u_{1}, u_{0}, \cdots, u_{k}\right] .
\end{aligned}
$$

Therefore $F_{k}\left[u_{0}, \cdots, u_{k}\right]$ is symmetric in its arguments if they are in $\mathcal{P}_{k}^{0}(\Omega)$.

Lemma 5. For any $u_{0}, u_{1} \in \mathcal{P}_{k}^{0}(\Omega)$ and any $v_{2}, \cdots, v_{k} \in \mathcal{P}_{k}(\Omega)$,

$$
F_{k}\left[u_{0}, u_{1}, v_{2}, \cdots, v_{k}\right]^{2} \leq F_{k}\left[u_{0}, u_{0}, v_{2}, \cdots, v_{k}\right] F_{k}\left[u_{1}, u_{1}, v_{2}, \cdots, v_{k}\right] .
$$

Proof. For $t \in \mathbb{R}$, consider the quadratic function

$$
\begin{aligned}
q(t) & =F_{k}\left[u_{0}+t u_{1}, u_{0}+t u_{1}, v_{2}, \cdots, v_{k}\right] \\
& =F\left[u_{1}, u_{1}, v_{2}, \cdots, v_{k}\right] t^{2}+2 F\left[u_{0}, u_{1}, v_{2}, \cdots, v_{k}\right] t+F\left[u_{0}, u_{0}, v_{2}, \cdots, v_{k}\right] .
\end{aligned}
$$

Since

$$
\begin{aligned}
q(t) & =\int_{\Omega}-\left(u_{0}+t u_{1}\right)\left(\sqrt{-1} \partial \bar{\partial} u_{0}+t u_{1}\right) \wedge\left(\sqrt{-1} \partial \bar{\partial} v_{2}\right) \wedge\left(\sqrt{-1} d d b v_{k}\right) \wedge \omega^{n-k} \\
& =\int_{\Omega} \sqrt{-1} \partial\left(u_{0}+t u_{1}\right) \wedge \bar{\partial}\left(u_{0}+t u_{1}\right) \wedge\left(\sqrt{-1} \partial \bar{\partial} v_{2}\right) \wedge\left(\sqrt{-1} d d b v_{k}\right) \wedge \omega^{n-k} \\
& \geq 0
\end{aligned}
$$

so

$$
F_{k}\left[u_{0}, u_{1}, v_{2}, \cdots, v_{k}\right]^{2} \leq F_{k}\left[u_{0}, u_{0}, v_{2}, \cdots, v_{k}\right] F_{k}\left[u_{1}, u_{1}, v_{2}, \cdots, v_{k}\right] .
$$

With Lemma 5 , both statements in Theorem 1 follow from the following algebraic lemma.

Lemma 6. Let $S$ be a set and $f$ be a nonnegative symmetric function defined on $S^{k}=S \times \cdots \times S$. Assume that for any $x_{1}, \cdots, x_{k} \in S$,

$$
f\left(x_{1}, x_{2}, x_{3}, \cdots, x_{k}\right)^{2} \leq f\left(x_{1}, x_{1}, x_{3}, \cdots, x_{k}\right) f\left(x_{2}, x_{2}, x_{3}, \cdots, x_{k}\right) .
$$

Then it follows that

$$
f\left(x_{1}, x_{2}, \cdots, x_{k}\right)^{k} \leq \prod_{j=1}^{k} f\left(x_{j}, \cdots, x_{j}\right) .
$$

Moreover if $S$ is a cone in some linear spaces and $f$ is linear in each of its arguments, then

$$
f(x+y, \cdots, x+y)^{1 / k} \leq f(x, \cdots, x)^{1 / k}+f(y, \cdots, y)^{1 / k}
$$

for any $x$ and $y$ in $S$.

The proof of this lemma can be found in Hör94.

To prove Theorem 2, we need another simple algebraic lemma for symmetric functions. 
Lemma 7. Let $\mu \in \Gamma_{k} \subset \mathbb{R}^{n}$ with $S_{k}(\mu)>0$. Then there exists $C$ depending only on $\operatorname{dist}\left(\mu, \partial \Gamma_{k}\right)$ such that for a positive integer $m<k$ and any $\lambda \in \Gamma_{k}$,

$$
S_{m}(\lambda) \leq C \tilde{S}_{k}(\lambda, \cdots, \lambda, \mu, \cdots, \mu),
$$

where $\lambda$ and $\mu$ appear $m$ and $k-m$ times in $\tilde{S}_{k}$ respectively.

Proof. Since $S_{k}(\mu)>0, \mu$ is in the interior of $\Gamma_{k}$. Therefore there exists $\varepsilon>0$ such that

$$
\mu-\varepsilon \mathbf{e} \in \Gamma_{k},
$$

where $\mathbf{e}=(1, \cdots, 1) \in \mathbb{R}^{n}$. Hence by Gårding's Inequality,

$$
\begin{aligned}
\tilde{S}_{k}^{\frac{1}{k-m}}(\lambda, \cdots, \lambda, \mu, \cdots, \mu) \geq & \tilde{S}_{k}^{\frac{1}{k-m}}(\lambda, \cdots, \lambda, \mu-\varepsilon \mathbf{e}, \cdots, \mu-\varepsilon \mathbf{e}) \\
& \quad+\tilde{S}_{k}^{\frac{1}{k-m}}(\lambda, \cdots, \lambda, \varepsilon \mathbf{e}, \cdots, \varepsilon \mathbf{e}) \\
\geq & \tilde{S}_{k}^{\frac{1}{k-m}}(\lambda, \cdots, \lambda, \varepsilon \mathbf{e}, \cdots, \varepsilon \mathbf{e})=\varepsilon S_{m}^{\frac{1}{k-m}}(\lambda) .
\end{aligned}
$$

Therefore

$$
S_{m}(\lambda) \leq \frac{1}{\varepsilon^{k-m}} \tilde{S}_{k}(\lambda, \cdots, \lambda, \mu, \cdots, \mu) .
$$

Proof of Theorem 2. Since $\Omega$ is strongly $k$-pseudoconvex, it is known Vin88, Li04 that there exists a unique $v \in \mathcal{P}_{k}^{0}(\Omega)$ such that $H_{k}[v]=1$. Hence there exists $\varepsilon>0$ depending on $v$ only such that $\operatorname{Hess}_{\mathbb{C}}(v)-\varepsilon I_{n} \in \Gamma_{k} \subset \mathcal{H}(n)$ pointwise in $\Omega$, where $I_{n}$ is the identity matrix of size $n$. Similar to the proof of Lemma 7 , there exists $C$ such that

$$
H_{m}[u]=S_{m}\left[\operatorname{Hess}_{\mathbb{C}}(u)\right] \leq C \tilde{S}_{k}\left[\operatorname{Hess}_{\mathbb{C}}(u), \cdots, \operatorname{Hess}_{\mathbb{C}}(u), \operatorname{Hess}_{\mathbb{C}}(v), \cdots, \operatorname{Hess}_{\mathbb{C}}(v)\right],
$$

where $\operatorname{Hess}_{\mathbb{C}}(u)$ and $\operatorname{Hess}_{\mathbb{C}}(v)$ appear $m$ and $k-m$ times in $\tilde{S}_{k}$. Therefore

$$
\begin{aligned}
I_{m}[u] & =\int_{\Omega}(-u) H_{m}[u] \\
& \leq C \int_{\Omega}(-u) \tilde{S}_{k}\left[\operatorname{Hess}_{\mathbb{C}}(u), \cdots, \operatorname{Hess}_{\mathbb{C}}(u), \operatorname{Hess}_{\mathbb{C}}(v), \cdots, \operatorname{Hess}_{\mathbb{C}}(v)\right] \\
& =C F_{k}[u, \cdots, u, v, \cdots, v] \\
& \leq C\left(I_{k}[u]\right)^{\frac{m+1}{k+1}}\left(I_{k}[v]\right)^{\frac{1}{k+1}} .
\end{aligned}
$$

So

$$
\left(I_{m}[u]\right)^{\frac{1}{m+1}} \leq \tilde{C}\left(I_{k}[u]\right)^{\frac{l+1}{k+1}}
$$

\section{Real Hessian integrals}

To prove Theorem 3 and Theorem 4 , we only need to show that the functional $G_{k}$ is symmetric in its arguments when they vanish on the boundary and the analogous Lemma 5 for $G_{k}$. They both follow from the divergence structure of polarized real Hessian operators.

Let $A^{(j)}, j=1,2, \cdots, k$ be symmetric $n \times n$ matrices. Then the polarized symmetric function $\tilde{S}_{k}$ is given by

$$
\tilde{S}_{k}\left(A^{(1)}, \cdots, A^{(k)}\right)=\frac{1}{k !} \sum_{\substack{i_{1}, \cdots, i_{k} \\ j_{1}, \cdots, j_{k}}} \delta_{j_{1} \cdots j_{k}}^{i_{1} \cdots i_{k}} A_{i_{1} j_{1}}^{(1)} \cdots A_{i_{k} j_{k}}^{(k)},
$$


where $\delta_{j_{1} \cdots j_{k}}^{i_{1} \cdots i_{k}}$ is the generalized Kronecker delta symbol. Denote

$$
\tilde{S}_{k-1}^{i j}\left(A^{(1)}, \cdots, A^{(k-1)}\right)=\frac{\partial}{\partial A_{i j}^{(k)}} \tilde{S}_{k}\left(A^{(1)}, \cdots, A^{(k)}\right) .
$$

Then

$$
\tilde{S}_{k}\left(A^{(1)}, \cdots, A^{(k)}\right)=A_{i j}^{(k)} \tilde{S}_{k-1}^{i j}\left(A^{(1)}, \cdots, A^{(k-1)}\right)
$$

and

$$
\tilde{S}_{k-1}^{i j}\left(A^{(1)}, \cdots, A^{(k-1)}\right)=\frac{1}{k !} \sum_{\substack{i_{1}, \cdots, i_{k-1} \\ j_{1}, \cdots, j_{k-1}}} \delta_{j_{1} \cdots j_{k-1} j}^{i_{1} \cdots i_{k-1} i} A_{i_{1} j_{1}}^{(1)} \cdots A_{i_{k-1} j_{k-1}}^{(k-1)} .
$$

Lemma 8. Let $u_{1}, \cdots, u_{k-1} \in \mathcal{C}^{2}(D)$. Then for any index $i \in\{1, \cdots, n\}$,

$$
\sum_{j=1}^{n} \frac{\partial}{\partial x_{j}} \tilde{S}_{k-1}^{i j}\left(\operatorname{Hess}\left(u_{1}\right), \cdots, \operatorname{Hess}\left(u_{k-1}\right)\right)=0 .
$$

Proof. Denote Hess $\left(u_{m}\right)=A^{(m)}, m=1, \cdots, k-1$. Then

(9)

$$
\begin{aligned}
& \sum_{j} \frac{\partial \tilde{S}_{k-1}^{i j}}{\partial x_{j}}=\frac{1}{k !} \sum_{j} \frac{\partial}{\partial x_{j}}\left(\sum_{\substack{i_{1}, \cdots, i_{k-1} \\
j_{1}, \cdots, j_{k-1}}} \delta_{j_{1} \cdots j_{k-1} j}^{i_{1} \cdots i_{k-1} i} A_{i_{1} j_{1}}^{(1)} \cdots A_{i_{k-1} j_{k-1}}^{(k-1)}\right) \\
& \quad=\frac{1}{k !} \sum_{\substack{i_{1}, \cdots, i_{k-1} \\
j_{1}, \cdots, j_{k-1}, j}} \delta_{j_{1} \cdots j_{k-1} j}^{i_{1} \cdots i_{k-1} i} \sum_{m=1}^{k-1} A_{i_{1} j_{1}}^{(1)} \cdots A_{i_{m-1} j_{m-1}}^{(m-1)} A_{i_{m} j_{m}, j}^{(m)} A_{i_{m+1} j_{m+1}}^{(m+1)} \cdots A_{i_{k-1} j_{k-1}}^{(k-1)} .
\end{aligned}
$$

Notice that $j_{m}$ and $j$ are anti-symmetric in the Kronecker symbol, while symmetric in $A_{i_{m} j_{m}, j}^{m}$, so the sum in equation (9) must be zero.

Once Lemma 8 is proved, then for any $k$-convex functions $v_{2}, \cdots, v_{k}$, and $\mathcal{C}^{2}$ functions $u_{0}$ and $u_{1}$ with $u_{0}=u_{1}=0$ on $\partial D$,

$$
\begin{aligned}
G_{k}\left[u_{0}, u_{1}, v_{2}, \cdots, v_{k}\right] & =\int_{D}-u_{0} \tilde{S}_{k}\left[\left(u_{1}\right)_{i j},\left(v_{2}\right)_{i j}, \cdots,\left(v_{k}\right)_{i j}\right] \\
& =\int_{D}-u_{0}\left(u_{1}\right)_{i j} \tilde{S}_{k-1}^{i j} \\
& =\int_{D}\left(u_{0}\right)_{i}\left(u_{1}\right)_{j} \tilde{S}_{k-1}^{i j}
\end{aligned}
$$

So $G_{k}$ is symmetric in its arguments if they vanish on the boundary, and moreover

$$
G_{k}\left[u, u, v_{2}, \cdots, v_{k}\right]=\int_{D} u_{i} u_{j} \tilde{S}_{k-1}^{i j} \geq 0
$$

for any $k$-convex $v_{2}, \cdots, v_{k}$, and $\mathcal{C}^{2}$ function $u$ with $u=0$ on $\partial D$. Then the same argument as in the proof of Lemma 5 can be used to show the following lemma, which implies Theorem 3 and Theorem 4 .

Lemma 9. For any $k$-convex functions $v_{2}, \cdots, v_{k}$, and any $\mathcal{C}^{2}$ functions $u_{0}$ and $u_{1}$, if $u_{0}=u_{1}=0$ on $\partial D$, then

$$
G_{k}\left[u_{0}, u_{1}, v_{2}, \cdots, v_{k}\right]^{2} \leq G_{k}\left[u_{0}, u_{0}, v_{2}, \cdots, v_{k}\right] G_{k}\left[u_{1}, u_{1}, v_{2}, \cdots, v_{k}\right] .
$$




\section{REFERENCES}

[Bak83] Ilya J. Bakelman. Variational problems and elliptic Monge-Ampère equations. J. Differential Geom., 18(4):669-699 (1984), 1983. MR730922 (85h:58045)

[Bło05] Zbigniew Błocki. Weak solutions to the complex Hessian equation. Ann. Inst. Fourier (Grenoble), 55(5):1735-1756, 2005. MR2172278 (2006e:32042)

[CP97] Urban Cegrell and Leif Persson. An energy estimate for the complex Monge-Ampère operator. Ann. Polon. Math., 67(1):95-102, 1997. MR1455430(98i:32021)

[Går59] Lars Gårding. An inequality for hyperbolic polynomials. J. Math. Mech., 8:957-965, 1959. MR0113978 (22:4809)

[Hör94] Lars Hörmander. Notions of convexity, volume 127 of Progress in Mathematics. Birkhäuser Boston Inc., Boston, MA, 1994. MR1301332 (95k:00002)

[Li04] Song-Ying Li. On the Dirichlet problems for symmetric function equations of the eigenvalues of the complex Hessian. Asian J. Math., 8(1):87-106, 2004. MR2128299 (2006d:32057)

[Tso90] Kaising Tso. On a real Monge-Ampère functional. Invent. Math., 101(2):425-448, 1990. MR.1062970 (91i:35082)

[TW98] Neil S. Trudinger and Xu-Jia Wang. A Poincaré type inequality for Hessian integrals. Calc. Var. Partial Differential Equations, 6(4):315-328, 1998. MR1624292 (99d:58163)

[TW02] Neil S. Trudinger and Xu-Jia Wang. Hessian measures. III. J. Funct. Anal., 193(1):1-23, 2002. MR1923626 (2003i:35106)

[Vin88] Alvaro Vinacua. Nonlinear elliptic equations and the complex Hessian. Comm. Partial Differential Equations, 13(12):1467-1497, 1988. MR970153 (89m:35083)

[Wan94] Xu Jia Wang. A class of fully nonlinear elliptic equations and related functionals. Indiana Univ. Math. J., 43(1):25-54, 1994. MR1275451 (95f:35089)

[Wan09] Xu Jia Wang. The $k$-Hessian equation. In Geometric Analysis and PDEs, volume 1977 of Lecture Notes in Math., Springer, Dordrecht, 2009. MR2500526

Department of Mathematics, Columbia University, New York, New York 10027

E-mail address: hou@math.columbia.edu

Current address: 504 East 81st Street, \#2M, New York, New York 10028

E-mail address: Zuoliang.Hou@gmail.com 